\title{
Análisis sistémico-funcional de textos algebraicos: hacia un entendimiento de su naturaleza discursiva en la historia y algunas implicaciones en su enseñanza
}

\author{
Systemic-functional analysis of algebraic texts: towards an understanding \\ of their discursive nature in history and some implications in their teaching \\ Luis Alberto López-Acosta \\ Daniel Rodríguez-Vergara
}

\begin{abstract}
RESUMEN
En la matemática educativa el estudio del lenguaje algebraico se ha centrado principalmente en los aspectos semánticos y sintácticos de su simbolismo, por lo que poco se ha estudiado acerca de la relación entre el componente del lenguaje natural y el simbólico. Por ello, en este escrito reportamos un análisis multisemiótico sistémico-funcional del componente experiencial en textos algebraicos antiguos relevantes en la historia del álgebra. La relevancia de estos textos se determinó a través de un estudio histórico epistemológico previo con el que se buscó recuperar significados opacos en su didáctica actual que pudieran plantear elementos para ser considerados en la enseñanza y aprendizaje del lenguaje algebraico. Con base en algunas consideraciones epistemológicas, el sistema de transitividad de la Teoría Lingüística Sistémico-Funcional fue adaptado para estos fines, obteniendo que el simbolismo no es un recurso semiótico autónomo en estos textos, además de que los participantes y procesos dentro de estos refieren a objetos matemáticos que no se catalogan únicamente como numéricos. Estos resultados contrastan con la típica caracterización del álgebra como un lenguaje de símbolos.
\end{abstract}

Palabras clave: matemática educativa, lenguaje algebraico, análisis experiencial, enseñanza del lenguaje, multisemiosis.

\section{AbSTRACT}

In mathematics education the study of algebraic language has focused mainly on the semantic and syntactic aspects of its symbolism, so little has been studied about the relationship between the component of natural and symbolic languages. Therefore, in this paper we report a systemic-functional and multisemiotic analysis of the experiential component in ancient algebraic texts relevant to the history of algebra. The relevance of these texts was determined through a previous historical epistemological study that sought to recover less known concepts in their current didactics that could pose elements to be considered in the teaching and learning of algebraic language. Based on some epistemological considerations, the system of transitivity of the Systemic-Functional Linguistic Theory was adapted for these purposes, obtaining that symbolism is not an autonomous semiotic resource in these texts, besides that the participants and processes within these refer to mathematical objects that are not catalogued only as numerical. These results contrast with the typical characterization of algebra as a language of symbols.

Keywords: mathematics education, algebraic language, experiential analysis, language education, multisemiosis. 


\section{INTRODUCCIÓN}

En matemática educativa cada vez va tomado más fuerza la importancia del estudio del lenguaje matemático y su vinculación con el aprendizaje y quehacer matemáticos (ver Pimm, 1987; Morgan, 2006; Schleppegrell, 2007; Morgan, Craig, Schuette y Wagner, 2014; Moschkovich, Wagner, Bose, Rodrigues y Schütte, 2018). En particular, diversas investigaciones han declarado implícita o explícitamente la necesidad de promover una conciencia metalingǘstica (ver Pimm, 1987; Halliday, 1993; McGregor y Price, 1999; Drouhard y Teppo, 2004; Schleppegrell, 2007), entendiéndola como "la capacidad lingüística que permite a un usuario de un idioma reflexionar y analizar el lenguaje hablado o escrito" (McGregor y Price, 1999, p. 451). De acuerdo con algunos estudios, se dice que esta habilidad implica desde el uso inconsciente e implícito de los componentes lingüísticos hasta una reflexión sistemática sobre la lengua como objeto (Fontich, 2010; Pascual, 2013; Pimm, 1987), lo cual demanda de un conocimiento profundo e imprescindible sobre los modelos gramaticales de referencia (Pascual, 2013), puesto que su ausencia puede tener repercusiones negativas tanto en el desempeño académico de los estudiantes como en la instrucción (McGregor y Price, 1999; Fontich, 2010; Pascual, 2013).

En este sentido, reconocemos necesario entender al lenguaje matemático, no solo desde una concepción generalista, sino como un macrolenguaje que integra diversos tipos de sublenguajes -geométricos, algebraicos y trigonométricos, entre otros-. Estos sublenguajes muestran diferencias sustanciales en el tipo de situaciones, problemas, razonamientos y objetos matemáticos que abordan, determinando así una diversidad de funciones y usos al interior de este sistema global. La tabla 1 muestra que, así como existen diferentes registros correspondientes al lenguaje verbal, también existen diferentes subsistemas correspondientes al lenguaje matemático. En este sentido, asumimos que en cada uno de estos sublenguajes se presentan distintos elementos de la experiencia y, por tanto, diferentes tipos de significados, que no se

Luis Alberto López-Acosta. Profesor asociado de tiempo completo de la Facultad de Matemáticas de la Universidad Autónoma de Yucatán, México. Es maestro en Ciencias con especialidad en Matemática Educativa por el Centro de Investigación y de Estudios Avanzados del Instituto Politécnico Nacional. Entre sus publicaciones recientes se encuentra el capítulo de libro "Experiencias formativas de investigadores en el desarrollo de proyectos doctorales de matemática educativa" (2020). Es miembro de la Asociación de Lingüística Sistémico-Funcional de América Latina, del Comité Latinoamericano de Matemática Educativa y de la International Community of Emerging Researchers in Mathematics Education. Correo electrónico: alberto.lopez@correo. uady.mx. ID: https://orcid.org/0000-0002-2903-5413.

Daniel Rodríguez-Vergara. Profesor titular de tiempo completo del Departamento de Lingüística Aplicada, Escuela Nacional de Lenguas, Lingüística y Traducción, Universidad Nacional Autónoma de México (UNAM). Doctorado en Lingüística y maestría en Lingüística Aplicada por la UNAM, licenciatura en Lenguas Modernas por la Benemérita Universidad Autónoma de Puebla (BUAP). Es miembro del Sistema Nacional de Investigadores, Nivel 1. Imparte clases en el Posgrado en Lingüística de la UNAM. Correo electrónico: danielrv@unam.mx. ID: https://orcid.org/0000-0002-6550-8863. 
han estudiado sistemáticamente. Así, antes de poder proponer elementos específicos para desarrollar un componente metalingǘstico en el quehacer del aula, reconocemos fundamental un entendimiento y conocimiento robusto sobre la naturaleza gramatical y semántica de los distintos sublenguajes que integran la semiótica de las matemáticas, como el algebraico, en el cual nos hemos centramos en este trabajo.

Tabla 1. Instanciación del lenguaje matemático.

\begin{tabular}{|c|c|c|}
\hline Sistema & Lenguaje verbal & Lenguaje matemático \\
\hline $\begin{array}{l}\text { Subsistemas } \\
\text { (registros) }\end{array}$ & $\begin{array}{l}\text { Registro académico, periodístico, } \\
\text { financiero, etc. }\end{array}$ & $\begin{array}{l}\text { Geometría, álgebra, } \\
\text { trigonometría, etc. }\end{array}$ \\
\hline
\end{tabular}

Sobre el álgebra se han producido conocimientos de algunos de sus elementos gramaticales, principalmente características semánticas y de reglas formales respecto al simbolismo (Chico, 2018). En particular, se han identificado dificultades en estudiantes en el tránsito del lenguaje natural al simbólico en términos de la interpretación de las regularidades discursivas de enunciados matemáticos, así como en la traducción y comprensión de problemas de palabras (McGregor y Price, 1999), y en la manipulación de los símbolos algebraicos (Bednarz, Kieran y Lee, 1996, McGregor y Price, 1999; Harel, Fuller y Rabin, 2008).

Entre las pocas caracterizaciones sobre el lenguaje algebraico, más allá de su simbolismo, se encuentra la de Drouhard y Teppo (2004), en la cual se destaca su carácter multisemiótico, una de las características del lenguaje matemático en general destacado por otros autores como O'Halloran (2005), Schleppegrell (2007), Morgan et al. (2014), Morgan (2014), Moschkovich et al. (2018) y Doran (2018). En este trabajo se caracteriza al lenguaje algebraico como una articulación de tres recursos semióticos: lenguaje natural, escritos simbólicos algebraicos y representaciones compuestas algebraicas. No obstante, el recurso del lenguaje natural presenta menos consideraciones en comparación con el de los escritos simbólicos algebraicos. Sobre este primer componente se recuperan elementos genéricos del lenguaje matemático como, por ejemplo, su carácter objetivado producto de procesos discursivos metafóricos, con los que se convierten procesos en objetos (Pimm, 1987; Halliday, 1993; O'Halloran, 2005; Schleppegrell, 2007; Sfard, 2008; Morgan, 2014). Además comprende un léxico especializado que involucra no solo términos especializados sino también palabras provenientes del lenguaje cotidiano que adquieren ciertas restricciones en su uso matemático, aspecto que algunos autores asumen que es una de las fuentes más comunes de dificultades en su aprendizaje (ver Pimm, 1987; Morgan, 2014).

Con base en estas consideraciones se vio la necesidad de incorporar marcos y herramientas analíticas que permitieran expandir las caracterizaciones actuales respecto 
al lenguaje algebraico, para entender qué aspectos lo diferencian de otros lenguajes, intentando profundizar en su gramática más allá de su simbolismo. Es así como se encontró en la lingüística sistémico-funcional (de aquí en adelante LSF) y en trabajos de Kay O’Halloran $(2000,2005,2007)$ elementos que están contribuyendo a este objetivo para determinar tanto sus funciones ideacional, interpersonal y textual como su carácter multisemiótico. En este escrito nos limitamos a reportar algunos resultados de un análisis experiencial de distintos textos matemáticos de distintas épocas y fases de desarrollo del discurso algebraico, con el propósito de identificar las características que hacen singulares los significados expresados en estos. Estos resultados forman parte de un estudio más amplio que tiene como objetivo comprender la naturaleza epistemológica y lingüística-semiótica del lenguaje algebraico desarrollado por Viète y Descartes en el Renacimiento (ver López-Acosta, 2019).

\section{FUNDAMENTACIÓN TEÓRICA Y METODOLOGÍA}

Este trabajo proviene de una investigación que incorpora elementos de la Teoría Socioepistemológica (TS de aquí en adelante), centrada en los procesos de construcción social y de difusión institucional de conocimiento matemático (Cantoral, 2013), y de la LSF (Halliday, 1982), conocida también como sociosemiótica por considerar al lenguaje como un sistema de significados situados social y culturalmente, junto con los trabajos de Kay O’Halloran (2000, 2005, 2007), investigadora que ha ampliado esta teoría para el estudio del discurso matemático.

En una primera fase de la investigación se realizó un estudio histórico-epistemológico (EHE de aquí en adelante) -ver López-Acosta (2019)- en el que se abordaron las preguntas ¿cuál fue el desarrollo del álgebra?, ¿qué tipos de operatividad simbólica estuvieron presentes a lo largo de la historia?, ¿qué justificaba dicha operatividad? Esta fase se fundamenta en el reconocimiento de que los contenidos de saber designados como saberes a enseñar son sujetos a un conjunto de transformaciones adaptativas que los harán aptos para ser objetos de enseñanza -a propósito del proyecto social que conlleva la educación- (Chevallard, 2000), fenómeno que soslaya elementos propios de los saberes originales que devienen opacos en el quehacer escolar. Por ello, la TS plantea necesario realizar EHE para obtener hipótesis epistemológicas que recuperen la génesis de los saberes en términos de las prácticas y las circunstancias socioculturales que las condicionan para confrontar los modelos epistemológicos escolares producto de este fenómeno, que puedan robustecerse y así construir vías alternativas de tratamiento escolar orientadas al desarrollo del pensamiento matemático con consideraciones socioculturales.

Los resultados del EHE develaron que el uso de un simbolismo formal no es una condición definitoria del pensamiento algebraico (Rojano, 1996; Radford, 2001). 
Por lo tanto, desde este punto de vista, nos cuestionamos en torno a la naturaleza del quehacer algebraico más allá de su simbolismo y manipulación. De aquí nuestro interés en profundizar respecto a sus elementos lingüísticos y epistemológicos que pudieran estar opacos con relación al álgebra manifiesta en los textos algebraicos antiguos y que pudieran ser recuperados en su tratamiento didáctico. Asimismo, a partir del reconocimiento de la relevancia de algunos algebristas en términos de los cambios de paradigma en el desarrollo del álgebra -como los babilonios (626 a.C.-539 a.C.), Diofanto de Alejandría (siglo III), al-Khwârizmî (780-850), Girolamo Cardano (1501-1576), Rafael Bombelli (1526-1572), Johannes Buteo (1492-1564/1572), François Viète (1540-1603) y René Descartes (1596-1650)-, se realizó una selección de textos pertenecientes a estos autores que abordaran métodos y técnicas sofisticadas de resolución de ecuaciones y problemas. Se priorizaron textos que abordaran ecuaciones de grado mayor que dos para garantizar un nivel de abstracción en los textos. Se construyó así un corpus conformado por ocho textos, de los cuales dos se consultaron en su idioma original (italiano y latín), cuatro en su versión traducida al inglés y dos al español. A pesar de consultar versiones traducidas (debido a las limitaciones de dominio de los distintos idiomas antiguos de los textos, p. ej. griego, árabe, francés), durante el análisis fue necesario compararlas con los textos originales -ver López-Acosta (2019) para más detalles-.

Si bien las traducciones implican que no se trabajó con la gramática original, se considera que la traducción a la gramática actual muestra rasgos importantes en términos experienciales de cada uno de los textos originales, que proporcionan elementos relevantes respecto a los significados que caracterizan al discurso algebraico en su desarrollo. Los textos son los siguientes:

1. TBA (texto babilonio): traducción al inglés de Høyrup (1986, p. 450, y 2002, p. 52) del texto del problema babilonio BM 13901 \#1. En la traducción se emplea la notación anacrónica decimal para facilitar el seguimiento del procedimiento de resolución.

2. TDI (texto de Diofanto): traducción al inglés de Meskens (2010) del texto del problema 8 del segundo libro de La Arithmetica de Diofanto (s. f.).

3. TAL (texto de Al-Khwârizmî): traducción al español de Puig (2008, pp. 14-15) del problema quinto del apartado "de los seis problemas" del Libro conciso de cálculo de al-jabry al-muqâbala de al-Khwârizmî (s. f.).

4. TCA (texto de Cardano [1993, pp. 243-246]): traducción al inglés de T. Richard Witmer del problema VI del capítulo XXXIX del Ars Magna de Cardano (1545). Se empleó la notación simbólica anacrónica de Witmer para facilitar el seguimiento del procedimiento de resolución.

5. TBO (texto de Bombelli): texto original en italiano de L'Algebra de Bombelli (1572, pp. 279-280). 
6. TBU (texto de Buteo): texto original en latín de la Logistica [...] de Buteo (1559, pp. 190-191).

7. TVI (texto de Viète [1983, pp. 403-405]): traducción al inglés de T. Richard Witmer de la Proposición XVI de Supplementum Geometriae de Viète (1646).

8. TDE (texto de Descartes [1947, pp. 79-80]): traducción al español de Pedro Rossell Soler, sobre el ejemplo que propone Descartes (1637) en el Libro II de La Géométrie.

Por lo tanto, en esta fase de la investigación se realizó un primer acercamiento a estos textos a través de un análisis LSF de las metafunciones ${ }^{1}$ experiencial, lógica y textual que Halliday (1982) ha caracterizado para el lenguaje en general. La metafunción experiencial alude a la función del lenguaje para representar la experiencia interna y externa del individuo. La lógica refiere a la función del lenguaje para crear conexiones lógicas entre los significados y reflejar una unidad coherente. La textual destaca la función del lenguaje para organizar y dar textura al mensaje. El análisis de la metafunción experiencial, el cual atañe a este trabajo, se lleva a cabo a través del sistema de transitividad, el cual representa una configuración de la experiencia de los individuos, expresando procesos sobre lo que experimentan uno o más actores (persona, lugar u objeto), denominados participantes, que se encuentran aludidos bajo ciertas circunstancias ${ }^{2}$ (ubicación, extensión, causa, modo, acompañamiento, papel, perspectiva y asunto). En este sentido, trata sobre la configuración de las categorías semánticas Participantes, Procesos y Circunstancias que representan la experiencia a través de una cláusula. ${ }^{3}$

De acuerdo con Butt, Fahey, Feez y Spinks (2012), el sistema de transitividad está compuesto por seis tipos de procesos. Los materiales, que aluden al hacer y que responden a las preguntas “¿qué hizo X?” o “¿qué paso?”; los mentales, que codifican la experiencia cognitiva, como la percepción, la inclinación o el afecto; los relacionales, que se subdividen en identificativos -aquellos que "relacionan al participante con su identidad, rol o significado" (Butt et al, 2012, p. 58) - y atributivos -aquellos que "relacionan al participante con su característica principal o descripción" (Butt et al, 2012, p. 58); los conductuales, que refieren al comportamiento sicológico o fisiológico "producto de la conciencia, pero que se manifiesta en el hablante" (Herrero, 2016, p. 68); los verbales, que expresan el 'decir' y los intercambios semióticos de información (Herrero, 2016), y los existenciales, que codifican la existencia del ser.

En términos generales, los análisis en LSF consisten en la identificación de las categorías semánticas (Participantes, Procesos y Circunstancias) en las cláusulas y

1 En el estudio no se incluyó la metafunción interpersonal.

2 En este trabajo no presentamos las funciones específicas para las circunstancias (ver López-Acosta [2019] para la descripción completa).

3 La cláusula es la unidad mínima de significado en LSF y comprende al menos un verbo. 
sus respectivas funciones dependiendo del tipo de proceso involucrado. ${ }^{4}$ Aquí las representaremos con el apoyo de organizadores tabulares ${ }^{5}$ como el siguiente:

[1] TBA5

\begin{tabular}{lll}
$1 / 4$ & a $3 / 4$ & se añade: \\
\hline Participante & Participante & Proceso \\
\hline Alcance & Beneficiario & Material \\
\hline Simbólico: Numérico & Simbólico: Numérico & Retórico: Geométrico \\
\hline
\end{tabular}

Algunas de las funciones de participantes que aparecen en los ejemplos de este trabajo son los siguientes: al estar presentes procesos materiales: actor (quien realiza el proceso), meta ('cosa' afectada), alcance ('cosa' no afectada). Para procesos relacionales atributivos: portador (el participante al que se le asigna la característica), atributo (la característica). Para procesos relacionales identificativos: identificado (a quien se le asigna una identidad), identificador (la identidad). Para procesos mentales: perceptor (quien percibe o realiza el proceso), fenómeno (sobre aquello que se siente, piensa, quiere, agrada/desagrada o percibe).

Este análisis se vio en la necesidad de realizar adaptaciones respecto a la caracterización del sistema de transitividad por dos consideraciones. La primera fue que, dado que entre los textos del corpus no todos contaban con simbolismo, planteamos una distinción, hasta ahora no realizada en las investigaciones en LSF respecto al lenguaje matemático, entre participantes y procesos que fueran retóricos (aquellos representados en términos del lenguaje natural) y simbólicos (aquellos representados mediante el simbolismo). La segunda fue que a partir de consideraciones epistemológicas provenientes del EHE relativas a los distintos tipos de actividad algebraica de los autores de estos textos, se identificó que los "objetos matemáticos" referidos en los textos podían ser numéricos, algebraicos o geométricos, algo que con un análisis descontextualizado de su carácter epistemológico no es posible notar. Por lo tanto, esto nos condujo a distinguir entre los participantes y procesos numéricos, algebraicos, geométricos y otros (aquellos no aplicados sobre objetos matemáticos) para identificar la experiencia representada en las cláusulas. En particular, los procesos sobre objetos matemáticos, que aluden a operaciones aritméticas o algebraicas, los ubicamos dentro de los materiales y relacionales, pues aluden tanto al "hacer" aunque sobre "objetos cognitivos", así como a establecer atributos o identificaciones entre ellos.

4 Para una descripción más completa sobre las funciones de los participantes de acuerdo con el tipo de proceso consultar Butt, Fahey, Feez y Spinks (2012, pp. 52-63) y Halliday y Matthiessen (2014).

5 En la nomenclatura de las tablas el número entre paréntesis corresponde al número del ejemplo, las letras representan las iniciales del texto correspondiente, y los números que siguen representan el número de la cláusula en el texto. 
Estas dos consideraciones resultan relevantes por el hecho de que permiten profundizar en la semántica de los textos algebraicos, para robustecer las caracterizaciones de este tipo de lenguaje, más allá de su simbolismo, el cual consideramos que provoca una sobregeneralización respecto a la función y significados asociados a este tipo de lenguaje. Un ejemplo de estas consideraciones puede verse en la siguiente cláusula:

[2] TBU24

\begin{tabular}{lllll} 
También & multiplique & ${ }^{\wedge}$ USTED & {$[[11 B, 2 C[54]]$,} & por 2 \\
\hline Proceso & Participante & Participante & Circunstancia \\
\hline Material & Actor & Meta & \\
\hline Retórico: Algebraico & Retórico: Otro & Simbólico: Algebraico & \\
\hline
\end{tabular}

Esta cláusula tiene una carga simbólica importante puesto que los signos que representan las operaciones y la igualdad algebraica son totalmente arbitrarios pues no representan ninguna relación directa con el significado subyacente. Por ejemplo "," representa la adición (“+”), mientras que "[" representa la igualdad (“=”). La expresión $11 B, 2 C[54$ puede traducirse actualmente como $11 y+2 z=54$. Nótese que, si bien el proceso "multiplique" podría considerarse un proceso aritmético, este no es el caso aquí, puesto que se está aplicando a una ecuación algebraica, no a un número, razón por la cual debe catalogarse como un proceso algebraico. Nótese además que la expresión simbólica es una cláusula simbólica donde el signo "[” representa un proceso relacional identificativo, razón por la cual se encuentra incrustada, de acuerdo con la convención sistémico-funcional por medio de los corchetes "[[]]". Esto revela una construcción metafórica en términos intersemióticos en el sentido de O'Halloran (2005), toda vez que, en la cláusula del lenguaje natural, la cláusula simbólica está siendo empleada como un grupo nominal. Por otro lado, como en muchas cláusulas dentro del corpus, el participante elidido “^USTED” representa al actor del proceso, lo cual no corresponde a ningún objeto matemático. A continuación presentamos otro ejemplo:

[3] TBA3

\begin{tabular}{ll} 
La mitad de 1 & se rompe \\
\hline Participante & Proceso \\
\hline Alcance & Material \\
\hline Retórico: Geométrico & Retórico: Geométrico \\
\hline
\end{tabular}

Esta cláusula proviene de un texto sin simbolismo. Con base en la interpretación de Høyrup (1986, 2002), en esta cláusula se identifica que el participante "La mitad de 1" corresponde a un objeto geométrico que representa la mitad del área de un 
rectángulo de base uno y altura desconocida (anacrónicamente $x$ ). El proceso "se rompe" representa la acción de dividir el rectángulo a la mitad para poder mover cada una de las dos mitades del rectángulo y así formar un cuadrado completo (figura 1). El texto completo muestra los primeros vestigios del procedimiento para "completar el cuadrado", como actualmente le llamaríamos.
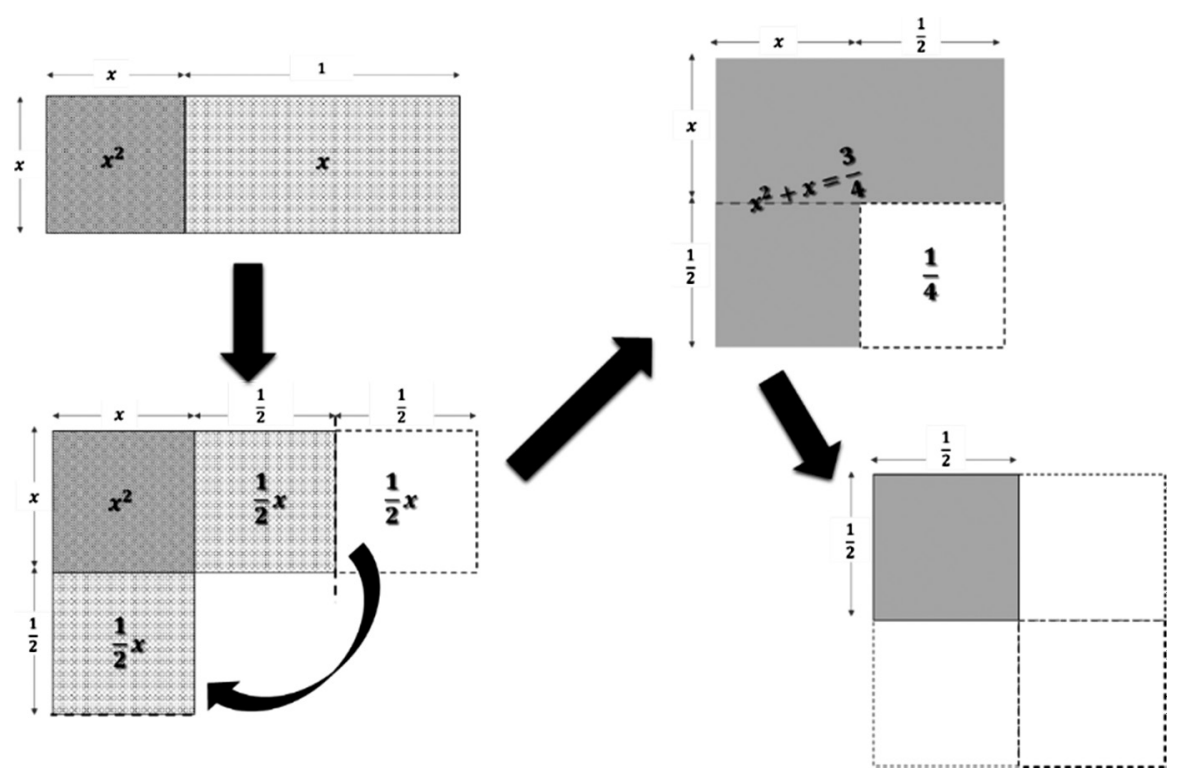

Figura1. Método figural geométrico subyacente en el texto.

Fuente: Adaptado de Radford (2001).

\section{Hallazgos}

A continuación mostramos ejemplos de textos analizados que presentan las características experienciales, centrándonos particularmente en los participantes (destacados en negritas) y procesos (destacados en cursiva). En el apartado 3.1 presentamos tres ejemplos de textos para ilustrar el tipo de análisis e ilustrar las características generales de estos. El primero texto (TAL) no recurre al simbolismo; el segundo (TBO) corresponde a una etapa casi simbólica, y el tercero (TDE) correspondiente a la etapa simbólica del álgebra. En el apartado 3.2 planteamos las características semánticas determinadas por el análisis de todo el corpus, dejando ver los tipos de procesos encontrados, así como la subdivisión de los procesos y participantes retóricos y simbólicos, y sus respectivas categorías como numéricos, algebraicos, geométricos y otros.

\section{Características generales de los textos}

La notación (C\#) hace referencia a la palabra cláusula y su respectivo número dentro del texto. El signo '[[ ]]' representa convencionalmente en la LSF una cláusula que está siendo empleada como un grupo nominal o adverbial al interior de otra, mientras 
que el signo ‘`’ es empleado para señalar la recuperación de los elementos elididos (en mayúsculas) en las cláusulas.

Texto de Al-Khwârizmî (TAL).

(C1) He dividido diez en dos partes; (C2) luego he multiplicado cada parte por sí misma (C3) y sumadas resultan cincuenta y ocho dirhams. (C4) Haces de una de las partes cosa (C5) y ${ }^{\wedge} H A C E S$ la otra diez menos la cosa. (C6) Multiplica luego diez menos cosa por sí mismo, (C7) resulta cien y un tesoro menos veinte cosas. (C8) Multiplica luego cosa por cosa, (C9) resulta tesoro. (C10) Suma luego ambos, (C11) resulta la suma cien y dos tesoros menos veinte cosas igual a cincuenta y ocho dirhams. (C12) Restaura luego esos cien y dos tesoros de las veinte cosas sustraídas (C13) y súmalas a los cincuenta y ocho, (C14) resulta [[ cien y dos tesoros igual a cincuenta y ocho dirhams y veinte cosas ]]. (C15) Reduce luego eso a un solo tesoro(C16) tomando la mitad del conjunto, (C17) resulta [[ cincuenta dirhams y un tesoro igual a veintinueve dirhams y diez cosas ]]. (C18) Opón luego con ése el otro, (C19) quitando veintinueve de cincuenta, (C20) queda [[ veintiún y tesoro igual a diez cosas ]]. (C21) Entonces balla la mitad de las raíces, (C22) resulta cinco; (C23) multiplica por sí mismo, (C24) resulta veinticinco. (C25) Quita luego de esto el veintiuno conectado con el tesoro, (C26) queda cuatro. (C27) Extrae luego su raíz, (C28) ^EL RESULTADO es dos. (C29) Quitala luego de la mitad de las raíces, [[ que es cinco ]], (C30) queda tres. (C31) Es una de las dos partes, (C32) y la otra es siete.

En este texto se muestra un ejemplo del método para resolver un problema de adición de dos números cuadrados cuya suma es una cantidad específica (58 en este caso). Las palabras 'tesoro', 'cosa', 'dirham', significaban respectivamente para al-Khwârizmî lo que actualmente entenderíamos como ' $x$ ' ', ' $x$ ' y 'constantes'. De tal suerte que la expresión inmersa en el texto corresponde a la expresión anacrónica: $x^{2}+(10-x)^{2}=58$. Hay dos tipos de participantes que predominan, los algebraicos y el propio autor o destinatario del texto (otros), los cuales se encuentran elididos en su mayoría. Los participantes numéricos aparecen al principio y al final del texto, puesto que, en términos de la estructura discursiva, las cláusulas de inicio (C1-C3) aluden a la relación numérica que se cumple y sobre la cual se desconocen las cantidades involucradas, mientras que en la parte final desde la cláusula 21 hasta la 32 se aplica una regla previamente demostrada para este tipo de problemas, de manera que se trabaja con las cantidades numéricas de la expresión. En el intermedio entre las cláusulas 4 y 20 el trabajo es sobre expresiones algebraicas. A continuación mostramos tres ejemplos [4-6].

[4] TAL1

\begin{tabular}{llll} 
He dividido & $\wedge$ YO & diez & en dos partes \\
\hline Proceso & Participante & Participante & Circunstancia \\
\hline Material & Actor & Alcance 1 & \\
\hline Retórico: Numérico & Retórico: Otro & Retórico: Numérico & \\
\hline
\end{tabular}


[5] TAL2

\begin{tabular}{|c|c|c|c|c|}
\hline luego & He multiplicado & ${ }^{\wedge} \mathrm{YO}$ & cada parte & por sí misma \\
\hline & Proceso & Participante & Participante & Circunstancia \\
\hline & Material & Actor & Alcance 1 & \\
\hline & Retórico: Numérico & Retórico: Otro & Retórico: Numérico & \\
\hline
\end{tabular}

[6] TAL12

\begin{tabular}{lllll} 
Restaura & $\wedge$ TÚ & luego & esos cien y dos tesoros & de las veinte cosas sustraídas \\
\hline Proceso & Participante & Circunstancia & Participante & Participante \\
\hline Material & Actor & Alcance & Fuente \\
\hline Retórico: Algebraico & Retórico: Otro & Retórico: Algebraico & Retórico: Algebraico \\
\hline
\end{tabular}

Texto de Bombelli (TBO).

(C1) Iguálese 16 p 36 a 20 3; (C2) restáurese 203 por parte. (C3) Lo que dará [ [1 6 p36 m20 3.p36 eguale az̧ero]]; (C4) por lo que la mitad del cubo, [[ que será m10]], agregándola al lado de 16, [[ que

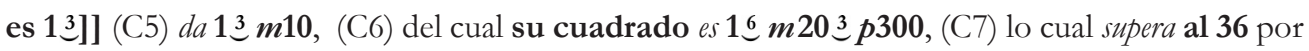
64. (C8) Pero agregando 64, a cada una de las partes (C9) hará [[1 6 m $m 203$ s p100 eguale a 64]], (C10) por lo que ${ }^{\wedge} C A L C U L A N D O$ el lado de ambas partes, (C11) se tendrá [[13 m10 eguale a 8]],

(C12) 16 p36 Eguale a 203

(C13) 16 m20 3 p36 Eguale a 0

(C14) 16 m20 3 3100 Eguale a 64

(C15) 13 m $m 10$ Eguale a 8

(C16) 13 Eguale a 18

(C17) 11 Eguale a R.c.18

(C18) Restaurando el negativo (C19) se tendrá [[13 Eguale a 18]], (C20) por lo que con el ^CALCULO DEL lado cúbico de ambas de las partes, se tendrá [[1_ Eguale a R.c.18]]. (C21) Pero la incógnita valdría R.c.18.

Este texto ejemplifica la forma de resolver una ecuación particular de grado seis que puede completarse como un cuadrado en la potencia cúbica. Esta ecuación puede traducirse anacrónicamente como $x^{6}+36=20 x^{3}$ (16 p 36 Eguale a 203 ). En Bombelli se dan rasgos casi totalmente simbólicos, puesto que el proceso 'Eguale $a$ ' se empleaba en el mismo sentido del signo actual ' $=$ ', mientras que ' 16 ' se corresponde con ' $x^{6}$ ', ' $p$ ' con ' + ' y '203 ' con '20 $x^{3}$ '. Se dice que es casi simbólico por el hecho de que los signos empleados por Bombelli consisten en abreviaciones de las palabras a las que refieren, a diferencia de una construcción simbólica como la mostrada previamente por Buteo. Puede verse que los participantes y procesos son casi en su totalidad sim- 
bólicos y algebraicos. Se muestra en [7] una excepción. Este texto en particular, por otro lado, es el único en todo el corpus que muestra cláusulas simbólicas (C12-C17) de manera independiente al texto. Sin embargo, nótese que repiten lo que en el texto completo se describe. Por lo tanto, representa rasgos similares a los de una ilustración que sintetiza el proceso de manera simbólica.

[7] $\mathrm{TBO} 2$

\begin{tabular}{lll} 
restáurese & 203 & por parte \\
\hline Proceso & Participante & Circunstancia \\
\hline Material & Alcance & \\
\hline Retórico: Algebraico & Simbólico: Algebraico & \\
\hline
\end{tabular}

En este ejemplo, el proceso 'restáurese' alude al hecho de efectuar la operación algebraica de 'sumar' el inverso aditivo de la expresión 203 para transformar la ecuación, de manera que resulte igualada a cero. Por tanto, se trata de un proceso para operar una entidad algebraica y no numérica. Por otro lado, el participante resulta ser simbólico y algebraico por ser un polinomio.

[8] TBO7

\begin{tabular}{llll} 
lo cual & supera & al 36 & por 64 \\
\hline Proceso & Participante & Circunstancia \\
\hline Relacional: Atributivo & Alcance & \\
\hline Retórico: Numérico & Retórico: Numérico & \\
\hline
\end{tabular}

En el caso de [8], superar al 36 por 64 significa que para que la expresión $16 \mathrm{~m} 203$ $p 36\left(x^{6}-20 x^{3}+36\right)$ sea un cuadrado perfecto en la potencia tres debe transformarse a $16 m 203 p 100\left(x^{6}-20 x^{3}+100\right)$; para ello es necesario agregar a cada lado de la ecuación lo faltante para que 36 sea 100, es decir, 64. De manera que estos objetos a los que se alude son números en la expresión.

Junto con Viète, Descartes es reconocido por haber dado el salto definitivo hacia el álgebra de las fórmulas simbólicas, la cual emplea sistemáticamente incógnitas/ variables (cantidades desconocidas) y parámetros (cantidades conocidas tratadas como desconocidas). Nótese que estas expresiones algebraicas no emplean números específicos como los antes discutidos, sino parámetros $a, b$ y $c$ por aplicarse no solo a relaciones numéricas sino también a relaciones geométricas entre figuras y líneas. Esto implica que muchos de los participantes correspondan a objetos geométricos. En particular, este es un ejemplo que usa Descartes para mostrar la forma con la que se puede determinar el género de una curva geométrica, la cual es construida por un compás (ver figura 2). A diferencia de los demás textos, este muestra una 
Texto de Descartes (TDE).

(C1) Como si quisiera saber (C2) de qué género es la línea $E C$ [[ que imagino descrita por la intersección de la regla $G L$ y la pieza $C N K L$, ]] (C3) cuyo lado $K N$ está prolongado indefinidamente hacia $C$, (C4) y que moviéndose ${ }^{\wedge} K N$ sobre el plano, en línea recta (C5) - es decir de tal manera que su lado $K L$ se encuentre siempre aplicado sobre alguna región de la línea $\boldsymbol{B} \boldsymbol{A}$ prolongada de uno y otro lado- (C6) bace mover circularmente la regla $G L$ alrededor del punto $G$, (C7) por estar ella vinculada (C8) de tal manera que ${ }^{\wedge} \mathbf{L A}$ REGLA pasa siempre por el punto $\boldsymbol{L}$. (C9) Elijo una línea recta [[ como $A B$ ]] (C10) para referir a sus diversos puntos todos los de la línea curva $E C$; (C11) y en esta línea $A B$ elijo un punto, [[ como el $\boldsymbol{A}]]$, (C12) para empezar por él el cálculo. (C13) Digo (C14) que elijo éste o aquella (C15) porque soy libre de tomarlos como quiera: (C16) pues aunque haya muchas maneras de elección (C17) para hacerla ecuación más corta y más fácil, (C18) siempre, $<<$ cualquiera sea la manera como se los tome, >> (C19) puede bacerse [[ que la línea aparezca de un mismo género ]], (C20) como es fácil demostrar. (C21) Después de esto, tomando un punto cualquiera de la curva, [[ como el $C$ ]], (C22) sobre el cual supongo (C23) que el instrumento [[ que sirve para describirla ]] está aplicado, (C24) trazoo por este punto $C$ la línea $C B$ paralela a la $G A$, (C25) y puesto que $\boldsymbol{C B}$ y $\boldsymbol{B} \boldsymbol{A}$ son dos cantidades indeterminadas y desconocidas, (C26) las designo a una y y a la otra $x$. (C27) Pero, para encontrar la relación de ambas, (C28) considero también las cantidades conocidas [[ que determinan el trazado de esa línea curva ]], tales como GA [[ que denomino $a$ ]]; $K l$, [[ que denomino $b$ ]] y $N L$ paralela a $G A$, [[ que denomino $c$ ]]. (C29) Luego digo: (C30) como $\boldsymbol{L N}$ es a $\mathbf{L K}$ (C31) o $\boldsymbol{c}^{\wedge} E S$ a $\boldsymbol{b}$, (C32) así $\boldsymbol{C B}$ [[ o sea $\boldsymbol{y}$ ]], es a $\boldsymbol{B K}$ [[ que es por consiguiente $\left.\frac{b}{c} \boldsymbol{y}\right]$ ]; (C33) y $\boldsymbol{B L}$ es $\frac{b}{c} \boldsymbol{y}-\boldsymbol{b}$; (C34) y $\boldsymbol{A L}$ es $\boldsymbol{x}+\frac{b}{c} \boldsymbol{y}-\boldsymbol{b}$. (C35) Además, como $\boldsymbol{C B}$ es a $\mathbf{L B}$ (C36) o $\boldsymbol{y}^{\wedge} E S a \frac{b}{c} \boldsymbol{y}-\boldsymbol{b}$, (C37) así $\boldsymbol{a}$ [[ o sea $\left.\left.\boldsymbol{G A}\right]\right]$ es a $\mathbf{L} \boldsymbol{A}$ o $\boldsymbol{x}+\frac{b}{c} \boldsymbol{y}-\boldsymbol{b}$. (C38) De manera que multiplicando la segunda por la tercera (C39) se obtiene $x+\frac{a b}{c} y-a b$, (C40) que es igual a $x y+\frac{b}{c} y y-b y$, (C41) que resulta multiplicando la primera por la última; (C42) y así que la ecuación [[ que se debía encontrar ]] es $\left[\left[\boldsymbol{y y}=\boldsymbol{c y}-\frac{c}{b} \boldsymbol{x y}+\boldsymbol{a y}-\boldsymbol{a c}\right.\right.$, $\left.]\right]$ (C43) En la cual se sabe (C44) que la línea EC es de primer género: (C45) pues, en efecto, ${ }^{\wedge}$ LA LÍNEA EC no es otra que una hipérbola.

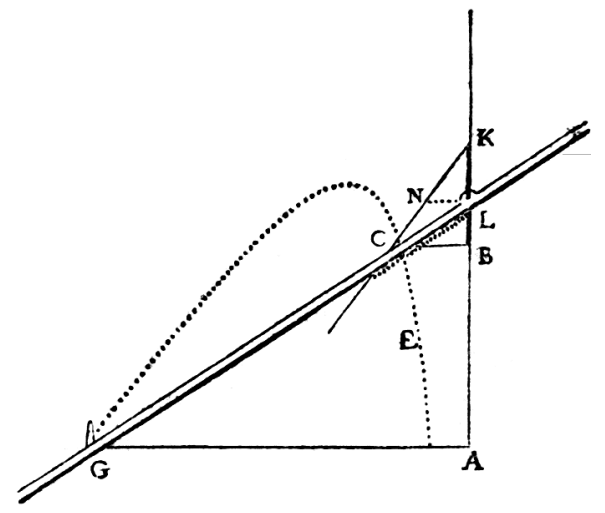

Figura 2. Compás referido en texto TDE.

Fuente: Descartes (1637, p. 321). 
predominancia del autor como participante, acompañado mayormente de procesos mentales o verbales. Por lo tanto, se identifica un significado experiencial centrado en la explicación del pensamiento y el procedimiento puesto en juego por el autor en la resolución (véase [9]).

[9] TDE1

\begin{tabular}{lll} 
como si & $\wedge$ & quisiera saber \\
\hline & Participante & Proceso \\
\hline Perceptor & Mental \\
\hline Retórico: Otro & Retórico: Otro \\
\hline
\end{tabular}

[10] TDE27

\begin{tabular}{|c|c|c|c|}
\hline Pero, & ${ }^{\wedge} \mathrm{YO}$ & encontrar & la relación de ambas \\
\hline & Participante & Proceso & Participante \\
\hline & Perceptor & Mental & Fenómeno \\
\hline & Retórico: Otro & Retórico: Otro & Retórico: Algebraico \\
\hline
\end{tabular}

En el caso de [10], el participante 'la relación de ambas' alude a la ecuación algebraica a la que se desea llegar para determinar el género de la curva. Por otro lado, en las cláusulas de [11-12] puede distinguirse que, si bien la configuración de participantes y procesos es en esencia la misma, sus significados son distintos.

[11] TDE30

\begin{tabular}{llll} 
como & LN & es a & LK \\
\hline Participante & Proceso & Participante \\
\hline Alcance 1 & Material & Alcance 2 \\
\hline Simbólico: Geométrico & Retórico: Geométrico & Simbólico: Geométrico \\
\hline
\end{tabular}

Aquí se da un proceso de determinación de una medida entre dos segmentos de recta representados por los símbolos $L N$ y $L K$. Así el proceso "es a" representa una división de las magnitudes de dichos segmentos, es decir, una razón geométrica dado que alude a la magnitud geométrica de los segmentos, a diferencia de [12].

[12] TDE31

\begin{tabular}{lll}
\hline$c$ & ${ }^{\wedge}$ ES a & $b$ \\
\hline Participante & Proceso & Participante \\
\hline Alcance 1 & Material & Alcance 2 \\
\hline Simbólico: Algebraico & Retórico: Algebraico & Simbólico: Algebraico \\
\hline
\end{tabular}


En este caso se involucra el mismo proceso de división que antes, solo que aplicado ahora a los representantes simbólicos de $L N$ y $L K$, que son $c$ y $b$ respectivamente, introducidos previamente en el texto. De modo que aquí el proceso está en un nivel semiótico distinto que el anterior, pues se aplica sobre los representantes simbólicos geométricos y no sobre los objetos geométricos propiamente, haciéndolo algebraico.

\section{Características semánticas de los textos}

A través del análisis sistémico-funcional se identificaron características globales del significado experiencial manifestado en todo el corpus. En la sección 3.2.1 se presentan los resultados concernientes a la frecuencia con la que fueron encontrados los distintos tipos de procesos: materiales, mentales, existenciales etc. Por otro lado, en la sección 3.2.2 se presentan resultados concernientes a la clasificación de los participantes y procesos en retóricos, simbólicos, numéricos (N.), geométricos (G.), algebraicos (A.) y de otra naturaleza (O.).

\section{Tipos de procesos en el corpus}

Con base en la tabla 2 puede identificarse que el discurso algebraico en estos textos tiene una predominancia respecto de los procesos relacionales, que representan $52 \%$ del total.

Esto indica que en esencia la experiencia está centrada en la determinación de relaciones entre los objetos matemáticos sobre los que se habla, sean estos números, polinomios u objetos geométricos, especificando sus características generales por medio de atributos ([13], descripciones [14], valores numéricos que corresponden a las incógnitas [15] y participantes algebraicos que pudieran ser geométricos o numé-

Tabla2. Frecuencia de procesos en el corpus.

\begin{tabular}{|c|c|c|c|c|c|c|}
\hline \multirow[b]{2}{*}{ Texto } & \multicolumn{6}{|c|}{ Procesos } \\
\hline & Existenciales & Materiales & Mentales & Rel. Atrib. & Rel. Ident. & Verbales \\
\hline $\mathrm{BA}$ & $22 \%$ & $56 \%$ & $0 \%$ & $0 \%$ & $22 \%$ & $0 \%$ \\
\hline DI & $0 \%$ & $30 \%$ & $10 \%$ & $30 \%$ & $20 \%$ & $10 \%$ \\
\hline $\mathrm{AL}$ & $32 \%$ & $47 \%$ & $0 \%$ & $6 \%$ & $15 \%$ & $0 \%$ \\
\hline CA & $6 \%$ & $17 \%$ & $6 \%$ & $28 \%$ & $39 \%$ & $6 \%$ \\
\hline $\mathrm{BO}$ & $29 \%$ & $24 \%$ & $0 \%$ & $19 \%$ & $29 \%$ & $0 \%$ \\
\hline $\mathrm{BU}$ & $11 \%$ & $29 \%$ & $5 \%$ & $13 \%$ & $39 \%$ & $3 \%$ \\
\hline VI & $7 \%$ & $5 \%$ & $5 \%$ & $49 \%$ & $33 \%$ & $2 \%$ \\
\hline $\mathrm{DE}$ & $9 \%$ & $36 \%$ & $11 \%$ & $31 \%$ & $7 \%$ & $7 \%$ \\
\hline Total & $13 \%$ & $26 \%$ & $5 \%$ & $27 \%$ & $25 \%$ & $3 \%$ \\
\hline
\end{tabular}

Nota. Las negritas destacan el tipo de proceso con mayor predominancia en cada texto. 
ricos [16-17]. En el caso de que el texto contenga simbolismo, como en [14], permite la adopción semiótica (O’Halloran, 2005), lo cual implica un tránsito entre los recursos semióticos de lenguaje natural y simbolismo.

[13] "y puesto que $\boldsymbol{C B}$ y $\boldsymbol{B} \boldsymbol{A}$ son dos cantidades indeterminadas y desconocidas", [TDE25]

[14] “cuyo lado KN está prolongado indefinidamente hacia C", [TDE3]

[15] "Pero la incógnita valdrá R.c.18”. [TBO21]

[16] [número] "Haces de una de las partes cosa" [TAL4]

[17] [líneas] "las designo a una y y a la otra $x$ ". [TDE26]

Por otro lado, los procesos relacionales identificativos tienen principalmente la función de preparar el significado experiencial algebraico, es decir, la operatividad con las expresiones y ecuaciones. Para ello, estos establecen equivalencias entre los participantes de manera que puedan aplicarse operaciones sobre el identificado o identificador para derivar nuevas expresiones [18-21], o bien para que en lo posterior en el texto se sustituyan los participantes no algebraicos por los algebraicos [22-24].

[18] Haces de una de las partes cosa [TAL4]

[19] $\mathrm{y}^{\wedge} \mathrm{H} A C E S$ la otra diez menos la cosa. [TAL5]

[20] Multiplica luego diez menos cosa por sí mismo, [TAL6]

[21] resulta cien y un tesoro menos veinte cosas". [TAL7]

[22] “у $\boldsymbol{B L}$ es $\frac{\boldsymbol{b}}{\boldsymbol{c}} \boldsymbol{y}-\boldsymbol{b}$ ” [TDE33]

[23] “Además, como $\boldsymbol{C B}$ es a $\mathbf{L B}$ ” [TDE35]

[24] “о $\boldsymbol{y} \wedge \mathrm{ES} a \frac{\boldsymbol{b}}{\boldsymbol{c}} \boldsymbol{y}-\boldsymbol{b}$ ”, [TDE36]

Los significados experienciales derivados de los procesos relacionales se complementan con los materiales, puesto que el trabajo algebraico desplegado se basa principalmente en la operatividad sobre las expresiones o ecuaciones, y de ahí que el siguiente grupo de procesos predominantes en el corpus sea de este tipo $(26 \%)$. Estos a su vez se complementan con los existenciales -terceros en presencia en el corpus $(13 \%)$ - puesto que cumplen la función de revelar los productos derivados de las transformaciones y operatividad sobre las expresiones o ecuaciones.

En menor cantidad los procesos mentales $(5 \%)$ y verbales $(3 \%)$ no tuvieron mucha presencia en el corpus. Esto coincide con lo que declara O'Halloran (2005) respecto a la limitada presencia de este tipo de procesos en el discurso matemático en general. En este corpus los textos de Diofanto y Descartes son los que contribuyeron 
a robustecer esta presencia, dado que ellos en particular muestran en sus textos una necesidad por "relatar" el procedimiento en términos de primera persona, aunque generalmente elidida, a diferencia de los otros textos.

\section{Categorías de participantes y procesos retóricos y simbólicos}

La tabla 3 presenta los porcentajes correspondientes a la aparición de participantes retóricos y simbólicos en cada texto. En ella se identifica que hay una predominancia de los participantes algebraicos simbólicos (35\%), lo cual podría deberse a las características del corpus, puesto que de los ocho textos solo dos no presentaban simbolismo. De haberse incluido otros textos previos a la época medieval, esto podría cambiar. Sin embargo, el corpus refleja justo lo encontrado en el EHE respecto al desarrollo progresivo del álgebra, mostrando una tendencia posterior a la cultura árabe a iniciar el tratamiento algebraico simbólico. A pesar de esto, puede notarse que los participantes retóricos (54\%) son predominantes en el corpus. Esto indica que aún estos textos algebraicos requieren del uso sistemático del lenguaje natural para poder comunicar su significado, es decir, el simbolismo algebraico no figura como un recurso semiótico autónomo en los textos. Incluso el único caso con complejos clausulares simbólicos es el del TBO, que como ya se ha mencionado, se presenta desconectado del texto completo y que además sintetiza el procedimiento descrito en este por medio de las ecuaciones asociadas.

Tabla 3. Frecuencia de tipos de participantes retóricos y simbólicos.

\begin{tabular}{|c|c|c|c|c|c|c|c|c|}
\hline \multirow[b]{3}{*}{ Texto } & \multicolumn{8}{|c|}{ Participantes } \\
\hline & \multicolumn{4}{|c|}{ Retóricos } & \multicolumn{4}{|c|}{ Simbólicos } \\
\hline & N. & G. & A. & O. & $\mathrm{N}$. & G. & A. & O. \\
\hline $\mathrm{BA}$ & $0 \%$ & $31 \%$ & $0 \%$ & $0 \%$ & $15 \%$ & $54 \%$ & $0 \%$ & $0 \%$ \\
\hline DI & $35 \%$ & $0 \%$ & $0 \%$ & $21 \%$ & $12 \%$ & $0 \%$ & $32 \%$ & $0 \%$ \\
\hline $\mathrm{AL}$ & $37 \%$ & $0 \%$ & $33 \%$ & $30 \%$ & $0 \%$ & $0 \%$ & $0 \%$ & $0 \%$ \\
\hline $\mathrm{CA}$ & $15 \%$ & $0 \%$ & $9 \%$ & $9 \%$ & $12 \%$ & $0 \%$ & $55 \%$ & $0 \%$ \\
\hline $\mathrm{BO}$ & $0 \%$ & $0 \%$ & $15 \%$ & $0 \%$ & $12 \%$ & $0 \%$ & $73 \%$ & $0 \%$ \\
\hline $\mathrm{BU}$ & $7 \%$ & $0 \%$ & $21 \%$ & $13 \%$ & $9 \%$ & $0 \%$ & $50 \%$ & $0 \%$ \\
\hline VI & $0 \%$ & $41 \%$ & $6 \%$ & $5 \%$ & $2 \%$ & $7 \%$ & $39 \%$ & $0 \%$ \\
\hline $\mathrm{DE}$ & $6 \%$ & $26 \%$ & $4 \%$ & $28 \%$ & $0 \%$ & $15 \%$ & $21 \%$ & $0 \%$ \\
\hline Total & $11 \%$ & $16 \%$ & $12 \%$ & $15 \%$ & $5 \%$ & $6 \%$ & $35 \%$ & $0 \%$ \\
\hline
\end{tabular}

Fuente: Construcción personal.

Los participantes que predominan en el corpus son los algebraicos, representando $47 \%$ del total, siguiendo los geométricos $(22 \%)$ y casi de manera equivalente los numéricos $(16 \%)$ y los de otra naturaleza $(15 \%)$. Respecto a estos últimos, principalmente 
los participantes refieren al autor o al destinatario del texto, lo cual refleja también que este tipo de discurso está pensado para mostrar una serie de procedimientos desde la visión del autor, los cuales tienen el propósito de replicarse por parte de los destinatarios (elididos en la mayor parte de los casos). Es decir, el significado experiencial trata en efecto con los objetos matemáticos (expresiones, ecuaciones, objetos geométricos y números) (85\%), pero también sobre la explicación que el autor hace del método que se sigue para enseñarle al destinatario (15\%).

Tabla 4. Frecuencia de tipos de procesos retóricos y simbólicos.

\begin{tabular}{|c|c|c|c|c|c|c|c|c|}
\hline \multirow[b]{3}{*}{ Texto } & \multicolumn{8}{|c|}{ Procesos } \\
\hline & \multicolumn{4}{|c|}{ Retóricos } & \multicolumn{4}{|c|}{ Simbólicos } \\
\hline & N. & G. & A. & O. & N. & G. & A. & O. \\
\hline $\mathrm{BA}$ & $0 \%$ & $44 \%$ & $0 \%$ & $56 \%$ & $0 \%$ & $0 \%$ & $0 \%$ & $0 \%$ \\
\hline DI & $0 \%$ & $0 \%$ & $45 \%$ & $50 \%$ & $0 \%$ & $0 \%$ & $5 \%$ & $0 \%$ \\
\hline $\mathrm{AL}$ & $21 \%$ & $0 \%$ & $32 \%$ & $47 \%$ & $0 \%$ & $0 \%$ & $0 \%$ & $0 \%$ \\
\hline CA & $0 \%$ & $0 \%$ & $39 \%$ & $33 \%$ & $0 \%$ & $0 \%$ & $28 \%$ & $0 \%$ \\
\hline $\mathrm{BO}$ & $0 \%$ & $0 \%$ & $28.5 \%$ & $43 \%$ & $0 \%$ & $0 \%$ & $28.5 \%$ & $0 \%$ \\
\hline $\mathrm{BU}$ & $0 \%$ & $0 \%$ & $66 \%$ & $18 \%$ & $0 \%$ & $0 \%$ & $16 \%$ & $0 \%$ \\
\hline VI & $0 \%$ & $12 \%$ & $17 \%$ & $58 \%$ & $0 \%$ & $0 \%$ & $13 \%$ & $0 \%$ \\
\hline $\mathrm{DE}$ & $0 \%$ & $22 \%$ & $7 \%$ & $71 \%$ & $0 \%$ & $0 \%$ & $0 \%$ & $0 \%$ \\
\hline Total & $3 \%$ & $9 \%$ & $29 \%$ & $49 \%$ & $0 \%$ & $0 \%$ & $10 \%$ & $0 \%$ \\
\hline
\end{tabular}

Fuente: Construcción personal.

Con respecto a los procesos, en la tabla 4 se muestra que la mayoría son de naturaleza retórica (90\%), y dentro de estos predomina más la categoría "Otra naturaleza" $(49 \%)$, los cuales en esencia refieren a procesos que no están aplicados propiamente a objetos matemáticos, sino que proveen de un marco para la experiencia algebraica, es decir, el establecimiento de relaciones e instrucciones como parte del método, así como de los productos de las acciones (véase [25-28]).

[25] “queda tres." [TAL30]

[26] "del cual su cuadrado es $1 \underbrace{6} m 2030$ s $p 100$," [TBO6]

[27] “y en esta línea $\boldsymbol{A B}$ elijo un punto, [[como el $\boldsymbol{A}]]$," [TDE11]

[28] “Pero, para encontrar la relación de ambas,". [TDE27]

En segundo lugar se encuentran los procesos retóricos algebraicos $(29 \%)$ con una marcada diferencia con los procesos simbólicos algebraicos (10\%), lo cual confirma que el simbolismo algebraico como recurso semiótico dentro del texto no alcanza 
una autonomía. La mayoría de las relaciones de equivalencia establecidas se hacen por medio del lenguaje natural. Incluso en el caso de Descartes, como representante de la última fase del desarrollo del álgebra simbólica, solo se encuentra una ecuación completamente simbólica que aparece incrustada como un grupo nominal dentro de una cláusula retórica. Como puede verse en [29-32], la mayoría de sus relaciones y operaciones algebraicas son realizadas por medio de recursos retóricos.

[29] “ De manera que multiplicando la segunda por la tercera [TDE38]

[30] se obtiene $\frac{a b}{c} y-a b$, que es igual a $x y+\frac{b}{c} y y-b y$ [TDE39-40]

[31] que resulta multiplicando la primera por la última [TDE41]

[32] y así que la ecuación [[ que se debía encontrar ]] es

$\left[\left[\boldsymbol{y y}=\boldsymbol{c y}-\frac{c}{b} \boldsymbol{x y}+\boldsymbol{a y}-\boldsymbol{a c},\right]\right] .[\mathrm{TDE} 42]$

Los procesos simbólicos algebraicos en estos textos corresponden únicamente a procesos relacionales, con los que se establecen relaciones de equivalencia entre participantes por medio del signo igual, el cual varía según el autor (véase [33-34]).

[33] “1 6 p36 Eguale a 20 3" [TBO12]

[34] " $y \boldsymbol{y}=\boldsymbol{c y}-\frac{c}{b} \boldsymbol{x y}+\boldsymbol{a y}-\boldsymbol{a c} "[$ TDE42]

\section{DisCUSIÓN Y CONCLUSIONES}

Planteamos dos consideraciones principales derivadas de este trabajo. La primera es que contrario a lo que escolarmente se suele pensar del álgebra, en tanto un tipo de discurso primordialmente simbólico, puede notarse aquí que el simbolismo no es un recurso semiótico autónomo en estos textos. Esto reafirma los resultados de algunos EHE que hemos mencionado previamente (Rojano, 1996; Radford, 2001), en torno a la idea de que no es necesario el simbolismo para dar muestra de pensamiento algebraico, como claramente se muestra en el texto TAL (sección 3.1.1). Sin embargo, la diferencia con estos estudios previos es que, por un lado, nuestros resultados abarcan no solo los textos totalmente retóricos o sincopados, ${ }^{6}$ a los que se suele referir para justificar la independencia del pensamiento algebraico respecto del simbolismo, sino también a aquellos representantes del álgebra simbólica como los de Viète y Descartes. Por otro lado, puesto que en estos estudios el foco es el pensamiento -en tanto analizan la relación entre el uso del simbolismo y su influencia en el despliegue de las

${ }^{6}$ Los textos sincopados se caracterizaron por introducir signos literales con la función de abreviar palabras recurrentes en el texto como los signos " $p$ ", “ $m$ ” y "R.c", en el caso del texto TBO, que abrevia las palabras italianas "più" (más), "meno" (menos) y "Radice cuba” (raíz cúbica) respectivamente. 
ideas, nociones y métodos algebraicos de resolución de problemas-, aquí abordamos la semiótica del texto, en tanto una instancia del lenguaje (Halliday y Mathiessen, 2014), extendiendo esta consideración hacia el texto, es decir, los textos algebraicos, aún los considerados en la fase simbólica, no recurren primordialmente al simbolismo para producir significados algebraicos robustos.

De este modo, estos resultados contrastan con definiciones de libros de texto y programas de estudio que plantean que "[El lenguaje algebraico] Expresa oraciones de lenguaje común en términos algebraicos [simbólicos]" (Colegio Nacional de Matemáticas, 2009, p. 270) y que es "un lenguaje que permite generalizar y expresar simbólicamente a los números y sus operaciones” (SEMS, s.f., p. 75), trayendo consigo reflexiones importantes respecto a la didáctica del álgebra, puesto que el significado detrás de estos textos va mucho más allá de la representación simbólica de un enunciado u objeto matemático. Podemos decir que el significado experiencial de los textos algebraicos analizados gira en torno al establecimiento de relaciones entre los distintos tipos de participantes, con la intención de conformar expresiones o ecuaciones que posteriormente serán sujetas a procesos materiales que deriven nuevos objetos que llevarán a la solución del problema, sea este numérico o geométrico. Esto se logra por medio de procesos que no se aplican sobre los objetos matemáticos per se pero que contextualizan el procedimiento y método llevado a cabo. Es decir, se identifica una intención didáctica y descripción del procedimiento del autor hacia un destinatario.

La segunda consideración es que los objetos matemáticos sobre los que se trabaja en estos textos no son únicamente números, como suele pensarse: "en álgebra se usan letras como $x, y, a, b$, y c para representar números" (Sullivan, 2006, p. 20); de hecho, al menos en estos textos, por debajo de los participantes algebraicos, hay mayor presencia de los geométricos. Esto contrasta también con lo planteado por Drouhard y Teppo (2004) respecto a la semántica de este lenguaje, argumentando que las expresiones algebraicas denotan funciones matemáticas. En López-Acosta (2019) se argumenta incluso que en términos epistemológicos en la última fase de desarrollo del álgebra simbólica por Viéte y Descartes fue la naturaleza geométrica de la actividad de estos dos matemáticos y no la aritmética lo que permitió la construcción de las ecuaciones paramétricas, las cuales emplean símbolos específicos para las incógnitas y parámetros.

Drouhard y Teppo (2004) plantean también que entre las dificultades del lenguaje algebraico se encuentra el hecho de que este hereda las características objetivadas del lenguaje matemático en general, producidas por nominalizaciones, lo cual pueden entenderse como casos de metáforas gramaticales (Halliday, 1998). Al menos en estos textos y muy probablemente durante toda esta fase de desarrollo del álgebra (250 a.C.-1637 d.C.) puede notarse que el discurso es muy congruente en este sentido. No obstante, a pesar de que una de las limitaciones del estudio fue no abordar la metáfora 
semiótica (O’Halloran, 2005), algunos de los textos sí presentan un carácter metafórico pero a nivel intersemiótico, el cual se da cuando en una cláusula retórica se incrusta la cláusula simbólica (ecuación simbólica) con la función de un grupo nominal, como la que hemos mostrado antes: "y así que la ecuación [[ que se debía encontrar ]] es [[ $\boldsymbol{y} \boldsymbol{y}=\boldsymbol{c} \boldsymbol{y}-\boldsymbol{c} / \boldsymbol{b} \boldsymbol{x y} \boldsymbol{+} \boldsymbol{a} \boldsymbol{y}-\boldsymbol{a c}, \mathrm{l}]$ ” (TDE, C42).

Así, incluso este tipo de construcción metafórica presente en algunos textos del corpus no coincide propiamente con una nominalización, ya que no se trata de un cambio de categoría gramatical en el lenguaje natural, pero sí de una cosificación (Sfard, 2008; Morgan, 2014), en tanto se convierte el proceso de "equivaler" (igualar dos expresiones) al objeto "equivalencia" (una ecuación). Por lo tanto, estos hallazgos amplían estas caracterizaciones sobre el lenguaje algebraico provenientes de las generalidades del lenguaje matemático actual, pues dejan ver otros aspectos profundos de este lenguaje que podrían permanecer ocultos de no analizarse con estas herramientas.

En este sentido, las herramientas analíticas proporcionadas por la LSF y el análisis multisemiótico, en conjunto con un entendimiento epistemológico de la actividad matemática y los recursos semióticos de los que se vale para producir significados, son elementos que consideramos primordiales para robustecer el entendimiento del lenguaje algebraico y matemático en general, para una didáctica que transite de lo monomodal a lo multimodal (Marín, 2017), coincidiendo con lo que menciona Doran (2018, p. 55): “[s]i buscamos desarrollar una pedagogía explícita que abarque la elaboración de significados multimodales, debemos tener ricas descripciones de los diversos recursos semióticos utilizados". De esta manera, esta visión epistemológicalingüística permitió reconocer elementos de adaptación del típico sistema de transitividad (Halliday y Matthiessen, 2014), los cuales resultan pertinentes para el estudio de los textos algebraicos y permiten profundizar en las características semánticas de estos, incorporando su carácter retórico, y sin restringirse al simbolismo. Así, el texto puede verse como una manifestación semiótica en la que confluyen distintas categorías de significados y objetos matemáticos que no corresponden únicamente a expresiones polinómicas o números, sino también a objetos geométricos, y la intención didáctica del autor por “enseñar" el método, aspectos gramaticales no señalados en las investigaciones previas respecto a este lenguaje.

Finalmente, en una fase posterior de la investigación que reportamos se sistematizará el análisis de las otras metafunciones y se llevará a cabo una experimentación con estudiantes para lograr una comprensión más robusta de las características gramaticales y multisemióticas del lenguaje algebraico, en tanto subsistema del lenguaje matemático como hemos argumentado en este trabajo, y sobre lo cual es necesario seguir profundizando. Eventualmente, estos análisis permitirán dilucidar elementos para propiciar una conciencia metalingüística desde una visión sociosemiótica en la enseñanza del lenguaje algebraico en particular y del matemático en general. 


\section{ReFERENCIAS}

Bednarz, N., Kieran, C., y Lee, L. (1996). Approaches to algebra: Perspectives for research. Dordrecht, Países Bajos: Kluwer.

Bombelli, R. (1572). L'algebra: parte maggiore dell'arimetica: divisa in tre libri. Bolonia: Giovanni Rossi.

Buteo, J. (1559). Logistica quae \& Arithmetica vulgò dicitur in libros quinque digesta ... ; eiusdem ad locum Vitruij corruptum restitutio, qui est de proportione lapidum mittendorum ad balistae foramen, libro decimo. En G. Rovillium. Lyon.

Butt, D., Fahey, R., Feez, S., y Spinks, S. (2012). Using functional grammar: An explorer's guide (3a. ed.). Victoria: Palgrave Macmillan.

Cantoral, R. (2013). Teoría socioepistemológica de la matemática educativa. Estudios sobre construcción social del conocimiento. México: Gedisa.

Cardano, G. (1993). Ars Magna or the rules of algebra (trad. T. Witmer). Nueva York: Dover Publications.

Chevallard, Y. (2000). La transposición didáctica, del saber sabio al saber enseñado (3a. ed.). Montevideo: Aiqué.

Chico, J. (2018). Impacto de la interacción en grupo en la producción de la lengua del álgebra en clase de matemáticas. Avances de Investigación en Educación Matemática, 14, 31-47.

Colegio Nacional de Matemáticas (2009). Matemáticas simplificadas (2a. ed.). México: Pearson Educación.

Descartes, R. (1637). Discours de la me'thode pour bien conduire sa raison \&o chercher la varite' dans les sciences plus la diotrique, les meteores, et la geometrie, qui sont des essais de cete methode. Leyden: Ian Marie.

Descartes, R. (1947). La geometría (trad. P. Rossell). Buenos Aires-México: Espasa-Calpe S.A.

Doran, Y. J. (2018). The discourse of physics: Building knowledge through language, mathematics and image. Nueva York: Routledge.

Drouhard, J.-P., y Teppo, A. (2004). Symbols and language. En K. Stacey, H. Chick y M. Kendal, The future of the teaching and learning of algebra (pp. 227-264). Boston: Kluwer Academic Publishers.

Fontich, X. (2010). La construcción del saber metalingüistic. Estudi sobre l'aprenentatge de la gramática d'escolars de secundària en el marc d'una seqüència didáctica [Tesis de Doctorado]. Barcelona: Universitat Autònoma de Barcelona, Departament de Didàctica.
Gascón, J. (1999). La naturaleza prealgebraica de la matemática escolar. Educación Matemática, 11(1), 77-88.

Halliday, M. A. (1982). El lenguaje como semiótica social: la interpretación social del lenguaje y el significado. México: Fondo de Cultura Económica.

Halliday, M. (1993). Some grammatical problems in scientific English. En M. A. Halliday y J. R. Martin, Writing science: Literacy and discursive power (pp. 69-85). London: Routledge.

Halliday, M. (1998). Language and knowledge: The unpacking of text. En J. Webster (ed.), The collected works of M. A. K. Halliday (pp. 24-48). Londres, Nueva York: Continuum.

Halliday, M., y Matthiessen, C. (2014). An introduction to functional grammar (4a. ed.). Nueva York: Routledge.

Harel, G., Fuller, E., y Rabin, J. (2008). Attention to meaning by algebra teachers. The Journal of Mathematical Behavior, 116-127.

Herrero Rivas, L. E. (2016). Transitividad y tipos de procesos en textos de historia de estudiantes y expertos en español. En N. Ignatieva y D. Rodríguez Vergara (eds.), Lingüistica sistémico funcional en México: aplicaciones e implicaciones (pp. 65-78). México: Universidad Nacional Autónoma de México.

Høyrup, J. (1986). Al-Khwarizmi, Ibn-Turk, and the Liber Mensurationum: on the origins of Islamic algebra. Erdem, 2(Ankara), 445-484.

Høyrup, J. (2002). Lengths, widths, surfaces: A portrait of old babylonian algebra and its kin. Heidelberg: Springer.

López-Acosta, L. A. (2019). Un acercamiento epistemológico y lingüistico para el estudio del pensamiento y lenguaje algebraico. El caso del análisis algebraico de Viète y Descartes [Memoria predoctoral no publicada]. México: Centro de Investigación y de Estudios Avanzados del Instituto Politécnico Nacional.

Marín, J. (2017). Enseñanza del lenguaje y nuevos alfabetismos; entre la tradición y la innovación. IE Revista de Investigación Educativa de la REDIECH, 7(10), 21-33.

McGregor, M., y Price, E. (1999). An exploration of aspects of language proficiency and algebra learning. Journal for Research in Mathematics Education, 30(4), 449-467. 
Meskens, A. (2010). Travelling mathematics - The fate of Diophantos' arithmetic. Basilea: Birkhäuser Verlag.

Morgan, C. (2006). What does social semiotics have to offer mathematics education research? Educational Studies in Mathematics, 61(1-2), 219-245.

Morgan, C. (2014). Mathematical language. En S. Lerman, Encyclopedia of mathematics education (pp. 388-391). Países Bajos: Springer.

Morgan, C., Craig, T., Schuette, M., y Wagner, D. (2014). Language and communication in mathematics education: An overview of research in the field. ZDM Mathematics Education, 46, 843-853.

Moschkovich, J., Wagner, D., Bose, A., Rodrigues, J., y Schütte, M. (eds.) (2018). Language and communication in mathematics education: International perspectives. Alemania: Springer.

O'Halloran, K. L. (2000). Classroom discourse in mathematics: A multisemiotic analysis. Linguistics and Education, 10(3), 359-388.

O'Halloran, K. L. (2005). Mathematical discourse: Language, symbolism and visual images. Londres-Nueva York: Continuum.

O'Halloran, K. (2007). Systemic functional multimodal discourse analysis (SF-MDA). Approach to mathematics, grammar and literacy. En A. McCabe, M. O'Donnell y R. Whittaker, Advances in language and education (pp. 75-100). Londres-Nueva York: Continuum.

Pascual, R. (2013). La actividad metalingüística en el aprendizaje de la lengua: consideraciones teóricas y aportes didácticos. En M. Guevara y K. Leyton (eds.), Enseñanza de la gramática (pp. 34-44). Mendoza: Facultad de Filosofía y Letras; Universidad del Cuyo; Sociedad Argentina de Lingüística.
Pimm, D. (1987). Speaking mathematically: Communication in mathematics classrooms. Lonres: Routledge \& Kegan Paul.

Puig, L. (2008). Componentes de una historia del álgebra. El texto de al-Khwarizmi restaurado. En Investigaciones en matemática educativa II (pp. 109-131). México: Grupo Editorial Iberoamérica. Recuperado de: https://www. uv.es/puigl/mexico96revisado03.pdf (consulta: 10 feb. 2019).

Radford, L. (2001). The historical origins of algebraic thinking. En R. Sutherland, T. Rojano, A. Bell y R. Lins, Perspectives on school algebra (pp. 13-63). Dordrecht: Kluwer.

Rojano, T. (1996). Developing algebraic aspects of problem solving within a spreadsheet environment. En N. Bednardz, C. Kieran y L. Lee, Approaches to algebra (pp. 137-146). Dordrecht: Kluwer.

Schleppegrell, M. (2007). The linguistic challenges of mathematics teaching and learning: A research review. Reading and Writting Quarterly, 23, 139-159.

Sfard, A. (2008). Thinking as communicating: Human development, the growth of discourse, and mathematizing. Cambridge: Cambridge University Press.

SEMS [Subsecretaría de Educación Media Superior] (s.f.). Bachillerato General. Recuperado de: http://www. sems.gob.mx/curriculoems/planes-de-estudio-dereferencia (consulta: 21 may. 2020).

Sullivan, M. (2006). Algebray trigonometría (7a. ed.). México: Pearson Educación.

Viète, F. (1646). Opera mathematica. Leiden.

Viète, F. (1983). The analytic art: Nine studies in algebra, geometry, and trigonometry from the Opus Restitutae Mathematicae Analyseos, seu, Algebrâ novâ by François Viète (trad. T. Witmer). Kent, Ohio: Kent State University.

Cómo citar este artículo:

López-Acosta, L. A., y Rodríguez-Vergara, D. (2021). Análisis sistémico-funcional de textos algebraicos: hacia un entendimiento de su naturaleza discursiva en la historia y algunas implicaciones en su enseñanza. IE Revista de Investigación Educativa de la REDIECH, 12, e1150. doi: 10.33010/ie_rie_rediech.v12i0.1150. 